\title{
Polytypism in cronstedtite; how various stacking sequences of layers affect diffraction pattern
}

\author{
Jiří Hybler \\ Institute of Physics of the Czech Academy of Sciences, Na Slovance 2, CZ-18224 Prague 8, Czech Republic \\ hybler@fzu.cz
}

The 1:1 layered silicate cronstedtite $\left(\mathrm{Fe}^{2+}{ }_{3-x} \mathrm{Fe}^{3+}{ }_{x}\right)\left(\mathrm{Si}_{2-x} \mathrm{Fe}^{3+}{ }_{x}\right) \mathrm{O}_{5}(\mathrm{OH})_{4}$, of the serpentine-kaoline group forms relative large amount of polytypes. They are subdivided into four OD subfamilies, or Bailey's groups A, B, C, D according to different stacking rules of identical (structure building) 1:1 layers (equivalents of OD packets) with trigonal protocell $a=5.5, c=7.1 \AA$. Distributions of so called subfamily reflections along the reciprocal lattice rows $[2 \overline{1} l]^{*} /[11 l]^{*} /[\overline{1} 2 l]^{*}$ in $\left(2 h \bar{h} l_{\text {hex }}\right)^{* /} /\left(h h l_{\text {hex }}\right)^{*} /\left(\bar{h} 2 h l_{\text {hex }}\right)^{*}$ planes of diffraction pattern is used for subfamily determination. Similarly, distributions of characteristic reflections along $[10 l]^{*} /[01 l]^{*} /[\overline{1} 1 l]^{*}$ rows in $\left(h 0 l_{\text {hex }}\right)^{*} /\left(0 k l_{\text {hex }}\right)^{*} /\left(\bar{h} h l_{\text {hex }}\right)$ planes allow determination of particular polytypes. For this purpose, graphical identification diagrams simulating distribution of reflections along named rows are used [1]. Owing modern diffractometers with area detectors and appropriate software, and/or Electron Diffraction Tomography (later EDT) technique, precession-like images of Reciprocal Space (later RS) sections corresponding to above listed planes can be easily and quickly obtained.

Lot of specimens of cronstedtite from various terrestrial localities and synthetic run products were studied by the author [1-5]. RS sections were recorded, and selected ones are presented in the lecture in order to demonstrate the variability of diffraction pattern.

In the subfamily $\mathrm{A}$, the stacking rule comprises $\pm \mathbf{a}_{\mathrm{i}} / 3$ shifts of consecutive layers. The most common is the $3 T$, relatively rare are $1 M$ and $2 M_{1}$ polytypes. They usually occur in $3 T+1 M, 3 T+2 M_{1}, 1 M+2 M_{1}$ allotwins. Monoclinic polytypes might be affected by twinning by reticular merohedry with $120^{\circ}$ rotation as twinning operation. Six-layer $6 T_{2}$ and three-layer triclinic $3 A$ polytypes are rare. Another possible twinning by $60^{\circ}$ rotation changes obverse setting of the subset of subfamily reflections into the reverse one $[1,4]$.

In the subfamily $\mathrm{D}$, the stacking rule is characterized by alternating $180^{\circ}$ rotations of consecutive layers, combined by $\pm \mathbf{b} / 3$ (of the orthohexagonal cell) or zero shifts. The most common polytypes are $2 \mathrm{H}_{1}$ and $2 \mathrm{H}_{2}$, occurring either isolated or in allotwins. Rarely, several six-layer polytypes were found. They usually occur in allotwins containing more polytypes, up to six! Diffraction patterns of such crystals are, of course, confusing. Fortunately, in many cases polytypes were isolated simply by cleaving crystals into smaller fragments, later studied separately. Hall et all. [6] derived 24 possible sequences of six-layer polytypes of subfamily D serpentines, valid also for cronstedtite. Their diffraction patterns were modelled, and compared with real RS sections. This simulation revealed, that five pairs of sequences (No. $4+6,7+18,8+10,9+13,11+12)$ provided identical diffraction patterns. Polytypes actually found correspond to following sequences: 1 (Hall's $\left.6 T_{1}\right), 5$ (proposed $\left.6 T_{3}\right), 8+10\left(6 T_{5}\right), 11+12\left(6 T_{4}\right), 24\left(6 T_{6}\right)$ (trigonal polytypes), $22\left(6 R_{1}\right)$, $23\left(6 R_{2}\right)$, (rhombohedral polytypes). The hexagonal polytype $6 H_{2}$ corresponding to the sequence 14 was also found. However, the identical diffraction pattern can be produced by the obverse-reverse twin of the rhombohedral polytype $6 R_{2}$ (sequence 23).

Allotwins of polytypes belonging to different subfamilies were rarely found. $1 M+1 T$ allotwins of subfamilies A and $\mathrm{C}$, respectively, was identified by EDT in the synthetic material [1]. The $\mathrm{C}$ subfamily is characterized by mere $\pm \mathbf{b} / 3$ or zero shifts, without any rotation. The allotwins of A+D subfamilies were found in some terrestrial samples. Sometimes, the A and D parts of such crystals were separated by cleaving into smaller fragments.

Many RS sections showed diffuse streaking of characteristic reflections along $\mathbf{c}^{*}$ due to partial stacking disorder. In extreme cases, reciprocal lattice rows are completely replaced by diffuse streaks.

The total number of ascertained polytypes of cronstedtite, recognized in RS sections, is 15 (+ one questionable).

[1] Hybler, J., Klementová, M., Jarošová, M., Pignatelli, I., Mosser-Ruck, R., \& Ďurovič, S. (2018). Clays and Clay Minerals 66, $379-402$.

[2] Hybler, J., Sejkora, J., \& Venclík, V. (2016). European Journal of Mineralogy, 28, 765-775.

[3] Pignatelli, I., Mugnaioli, E., Hybler, J., Mosser-Ruck, R., Cathelineau, M., \& Michau, N. (2013). Clays and Clay Minerals 61, $277-289$.

[4] Hybler, J., Števko, M., \& Sejkora, J. (2017). European Journal of Mineralogy, 29, 91-99.

[5] Hybler, J., Dolníček, Z., Sejkora, J., \& Števko, M., (2020). Clays and Clay Minerals 68, 632-645.

[6] Hall, S. H., Guggenheim, S., Moore, P., \& Bailey, S. W. (1976). Canadian Mineralogist 14, 314-321.

Keywords: cronstedtite; polytypism; layer stacking; X-ray diffraction; electron diffraction tomography; allotwins 